\title{
Methodology for metrological analysis of periodic coding images: measurement resolution and spatial resolution
}

\author{
C. Badulescu, M. Bornet, J.-C. Dupré, S. Equis, M. Grédiac, J. Molimard, P. Picart, R. Rotinat, \\ V. Valle
}

GDR CNRS 2519, « Mesure de champs et identification en mécanique des solides »

\begin{abstract}
Optical full-field techniques have a great importance in modern experimental mechanics. Even if they are reasonably spread among the university laboratories, their diffusion in industrial companies remains very weak for several reasons, especially a lack of metrological performance assessment. A full-field measurement can be characterized by its resolution, bias, measuring range, and by a specific quantity, the spatial resolution. The present communication proposes an original procedure for the estimation in one single step of the resolution, bias and spatial resolution for a given operator (decoding algorithm, such as image correlation, low-pass filters, derivation tools, ...). This procedure is based on the construction of a particular multi-frequential field, and a Bode diagram representation of the results. This analysis is applied to various phase demodulating algorithms for the estimation of in-plane displacements
\end{abstract}

\section{Introduction}

Full field optical methods are commonly used in experimental mechanics to obtain kinematical data. These methods are powerful tools, easy to use, but their metrological characteristics are not totally defined. The diversity of algorithms, used to analyse the acquired images, can perturb non-specialist users. Consequently, the diffusion of these methods in the industrial context is still limited. The GDR2519 group (Mesures de champs et identification en mécanique des solides) supported by the French CNRS, has for objective to clarify this situation. This group proposes benchmarks to analyse the performances of algorithms used in several optical methods. The current works are based on the algorithms allowing the phase extraction from fringe pattern. These algorithms are usually employed to obtain kinematical data from optical methods like DSPI method, grid method and moiré method. The aim of this work is to propose a metrological process which can define the performances of the different phase extraction techniques. First, the proposed procedure is to generate a pair of fringe patterns corresponding to the reference and the deformed mechanical states. The deformed image must contain complex displacement fields allowing the calculation of the metrological performances. In a second time, analysis tools are proposed to evaluate the measured displacement at each pixel of the image and to give performances of each specific extraction algorithms. Results are shown and discussed. 


\section{Displacement field and fringe pattern definition}

The proposed work is based on the analysis of fringe images which have been created from a known displacement $U_{y}$. To limit the number of generated images, we have chosen to analyse multiple frequency responses with only two images: A reference one and a deformed one. For the deformed image, we proposed to use a periodic displacement with a variable periodicity along the $y$ axis.

$$
U_{y}=A_{U} \times \cos \left(\frac{\pi}{P} \frac{y^{2}}{y_{\max }}\right)=A_{U} \times \cos (F)
$$

where $A_{U}$ is a constant amplitude, $y_{\max }$ is the image height and $P$ is the minimal wave length of the periodic displacement. This choice allows obtaining a linear evolution of the local frequency in function of $y$ :

$$
f_{y}=\frac{1}{2 \pi} \frac{\partial F}{\partial y}=\frac{1}{P} \frac{y}{y_{\max }}
$$

It can be noted that the minimal wave length of the created periodic displacement is reached at the bottom of the image. In order to separate the signal phase from the signal frequency, a term, corresponding to a linear evolution of the phase along the $x$ axis, has been added. The displacement can be written as :

$$
U_{y}=A_{U} \times \cos \left(\frac{\pi}{P} \frac{y^{2}}{y_{\max }}+2 \pi \frac{x}{x_{\max }}\right)
$$

Figure 1 corresponds to the displacement field obtained with $A_{U}=0,3, P=5, y_{\max }=1024$ et $x_{\max }=512$ (pixels) and used in the presented work.

For the first tests, a perfect sinusoidal modulation has been applied and can be expressed by the following mathematical representation of the image intensity.

$$
I=B_{I}\left[1+\frac{A_{I}}{B_{I}} \cos \left(2 \pi \frac{y+U_{y}}{P_{o p t}}\right)\right]
$$

where $A_{I}$ is the amplitude modulation and $B_{I}$ is the background illumination. $A_{I}$ and $B_{I}$ have been chosen equal to 127.5 gray levels and $P_{\text {opt }}$, the optical pitch, equal to $5,6,7$ and 8 pixels. For the presented test, the noise present in the real images has been neglected.

The reference image is built with $U_{y}=0$ and the deformed one with the expression (3).

\section{Results}

Pairs of images have been generated and analysed by the 7 partners (NAM, LAMI, LAUM, LMPF, LMS-P, LMS-X, LTDS) with their own processing tools, as will be describe later. Displacement fields are obtained for any points of the map (except eventually at the border of the images). They are analysed using the following procedure: for each line, i.e. for each spatial frequency, the best sine is extracted from experimental points using Fourier transform. Relative difference ErrA between the recorded amplitude and the theoretical amplitude $A_{U}$ is then given as a function of spatial frequency in a log-log scale (Fig. 2). In an ideal case, such a diagram presents two zones: first one, for the higher spatial frequencies, is described by a horizontal line $\operatorname{ErrA}=1$; this indicates that such frequencies are strictly unreachable with the given image processing. Second zone is a linear 
decreasing. The intersection between these two lines defines the ultimate spatial resolution (RSU) of the given method: no event with a size lower than this SRU can be detected. It is possible to define another practical quantity corresponding to the maximal allowed error, for example $10 \%$, denoted RS10\%. In the same way, the slope of the second part of the curve gives the filter order of the given operator, i.e. its ability to render without bias signals with length higher that the cutting wavelength.

Last interesting quantity is root mean square difference between measured signal and interpolated sine curve. It gives an indication on the random error $\sigma_{U}$ present in the signal. Again, it can be plotted as a function of spatial frequency; Fig. 2 shows an example

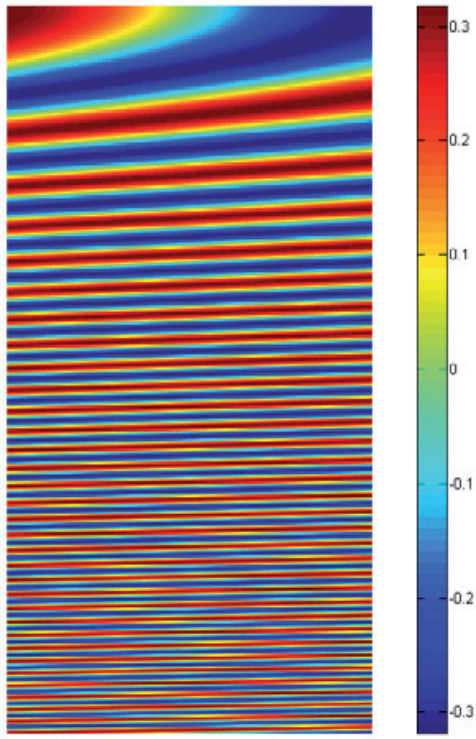

Fig.1. : Displacement fields (in pixels).

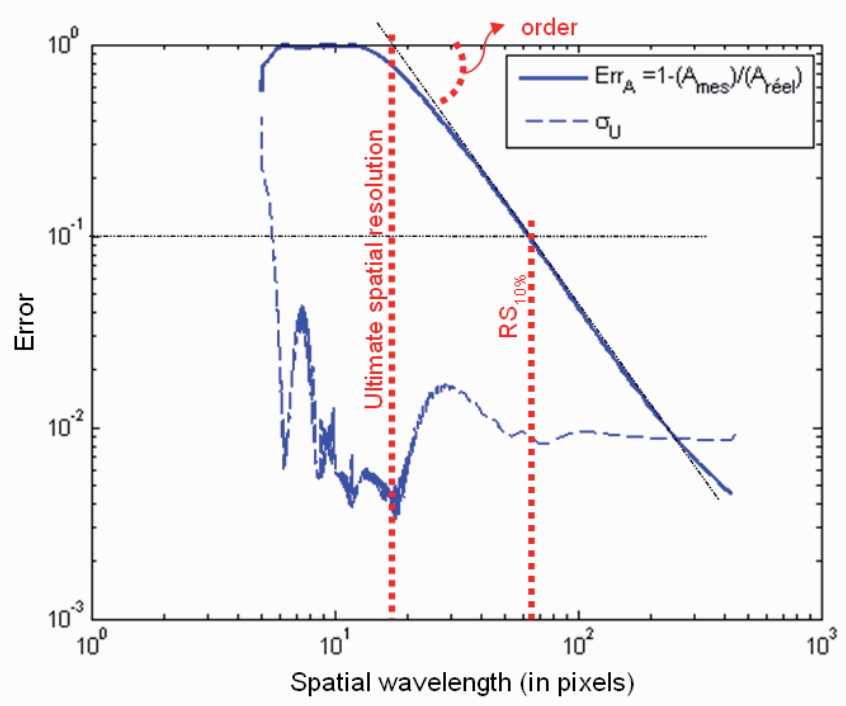

Fig.2. : Results analysis.

\section{Comparison between different analysis methods of image with spatial carrier}

In this work, various methods of treatment were used according to the usual practice of the laboratories:

\subsection{Spatial Fourier-transform (LAUM)}

Historically, the global spatial Fourier-transform was used in the first methods of demodulation [2]. In the Fourier spectrum, a spatial carrier signal is revealed as three amplitude peaks, one centered on zero, and two symmetrical peaks centered respectively over the wavelength $+p$ and the wavelength $-p$, where $p$ is the pitch of the carrier. The complex formulation of the intensity revealing the terms of modulation $+\mathrm{p}$ and $-\mathrm{p}$ is:

$$
\begin{aligned}
I(x, y)= & \frac{1}{2} A_{I}(x, y) \exp (i \varphi(x, y)) \exp \left(2 i \pi \frac{y}{p}\right) \\
& +\frac{1}{2} A_{I}(x, y) \exp (-i \varphi(x, y)) \exp \left(-2 i \pi \frac{y}{p}\right)+B(x, y)
\end{aligned}
$$

The phase of the signal is then given by: 


$$
\varphi(x, y)=\arctan \left(\frac{\Im(\hat{c})}{\mathfrak{R}(\hat{c})}\right) \text { with } \hat{c}=T F\left(\frac{1}{2} A_{I}(x, y) \exp (-i \varphi(x, y))\right)
$$

where $\hat{c}$ is one of the two peaks of the Fourier-transform, shifted in the frequency spectrum of the $-1 / p$ quantity. If the three peaks composing the signal are quite distinct, it is easy to select one of these peaks and to return zero to the remainder of the signal with a two-dimensional filter centered on the $1 / \mathrm{p}$ frequency. According to the width of the filter used, the phase extraction algorithm will be more or less filter. For this study, the width of the filter is remained constant; the position of the peak is adapted to each case.

\subsection{Windowed discrete Fourier-transform (LaMI, LMPF, LTDS)}

The phase extraction is performed by seeking the particular term argument of the local Fouriertransform of the intensity map. It is necessary to define an arbitrary pitch, by definition different from the pitch of grid, to demodulate the signal of phase. The formulation suggested by Y. Surrel [3] is written:

$$
\varphi=-\arctan \frac{\sum_{k=0}^{p-1} k\left(I_{k-1}-I_{2 \mathrm{p}-k-1}\right) \sin (2 \mathrm{k} \pi / p)}{p I_{p-1}+\sum_{k=0}^{p-1} k\left(I_{k-1}-I_{2 \mathrm{p}-k-1}\right) \cos (2 \mathrm{k} \pi / p)}
$$

where $p$ is the estimated pitch of the grid (in pixels), $k$ is the coordinate of the current point and $I_{k}$ is the local intensity of image. This formulation is in fact a mere convolution between a Fouriertransform of the signal and a triangular window. The implementation used in Laboratories supposes an adaptation of the pitch of coding to each situation (5-6-7 or 8 pixels per pitch). The width of the bi-triangular window used here is $2 \times p-1$ pixels.

\subsection{Spectral analysis (LMS-P (AS))}

Spectral analysis is based on the calculation of the discrete bidimensional Fourier transform of a zone of interest with a size of $\mathrm{N} \times \mathrm{N}$ pixels associated to an interpolation of the frequency spectrum along two directions in order to obtain the orientation of the grating [5] [6]. With this technique, periodicity of the signal $(\mathrm{p})$, initial phase $(\phi)$ and complex amplitude A can be calculated. They depend on the complex values of the principal peak of coordinate $K$, the Fourier-transform and the adjoining peaks. If $H_{k}$ are the complex values of the Fourier-transform of the signal, $\beta$ is defined such as [5]:

$$
\beta=R_{e}\left(\frac{H_{K-1}-H_{K+1}}{2 \mathrm{H}_{K}-H_{K+1}-H_{K-1}}\right)
$$

The pitch of the signal $p$, its complex amplitude and the phase are given by:

$$
\begin{aligned}
& p=\mathrm{N} /(\mathrm{K}+\beta) \text { with }-0,5<\beta<0.5 \\
& A=\frac{\left.j 2 \pi 2 \pi \beta^{2}-1\right)}{1-e^{j 2 \pi 2}}\left(2 \mathrm{H}_{K}-H_{K-1}-H_{K}\right)
\end{aligned}
$$




$$
\varphi=\arctan \frac{I_{m}(A)}{R_{e}(A)}
$$

In this work, dimensions of windows used for the spectral analysis are $32 \times 32$ pixels.

\subsection{DIC technique (LMS-X).}

Marking surface by a regular grid constitutes a particular way of suitable marking for an analysis per digital image correlation. The classical algorithms used in DIC require however some adaptations [4] to take account of the periodicity of such marker. Taking into account the definition of the field of displacement, the deformed image was selected like image of reference according to usual conventions in digital image correlation. A very simple algorithm of DIC, based on an assumption of uniform translation and a bilinear interpolation of the gray levels of the final image (i.e. not deformed in this case), was used with zone of interest of width 3 pixels (for practical reasons) and of height 3, 6 or 9 pixels. Let us note that this analysis does not make any assumption a priori on the marking form and its wavelength.

\subsection{Modulated phase correlation method (LMS-P (MPC))}

The principle of the MPC algorithm (Modulated Phase Correlation) lies in the adaptation of the correlation technique searches in a zone of interest for the degree of similarity between a real fringe pattern and a mathematical model [7].

Usually the mathematical representation of a two-dimensional fringe pattern is globally expressed by:

$$
I_{v}(x, y)=A(x, y) \cos [\Phi(x, y)]+B(x, y)
$$

where $A(x, y)$ is the amplitude modulation, $B(x, y)$ is the background illumination, and $\Phi(x, y)$ is the value of the phase. In this global representation $A, B$ and $\Phi$ are complicated functions that depend on coordinates $x$ and $y$. If we consider only a small zone of interest centered at coordinates $(x, y)$, the global expression can be reduced to a simpler one in which $\Phi$ depends on coordinates $(\xi, \gamma)$, and where $x$ and $y$ are fixed. Our mathematical model, representing fringes with constant pitch and orientation, is described by:

$$
I=A \cos [\Phi(\alpha, p, \varphi, \xi, \gamma)]+B
$$

Variables $p$ and $\alpha$ are, respectively, parameters of pitch and orientation of the fringe pattern, and $\varphi$ is the term of the demodulated phase. Indeed, $A$ and $B$ parameters are considered constant in this zone of interest.

For calculation of the degree of similarity between the mathematical expression designated $I$ and the real fringe pattern denoted $I_{r}$, we use the formulation

$$
\psi(A, B, \alpha, p, \varphi)=\iint_{D}\left(I_{r}(x, y)-I(x, y)\right)^{2} d s
$$

To minimize the $\Psi$ function we cannot use a gradient method because we have a periodic function that depends on $\alpha$ and $\varphi$. For this reason we must calculate all the values of $\Psi$ that correspond to all the possible values for each parameter $\alpha, p, A, B$, and $\varphi$. The minimum of $\Psi$ gives directly the five parameters. For the present study, the computation is made with dimensions of $8 \times 8$ and $16 \times 16$ pixels for the zone of interest. 


\subsection{First results}

First results are shown Fig. 3 5. They are obtained according to the common practice of each laboratory. Spectral methods, in red, correspond to the discrete Fourier Transform implemented at LAMI and LTDS. In the latter laboratory proposed two ways, with or without including a spatial filter of $2 \times p-1$ length. Last method in this group is spectral analysis (LMS-P (AS)). Correlation methods (in blue) are Modulated Phase Correlation (MPC), denoted LMS-P(MPC) or a special implementation of a digital image correlation code, with different windows: $3 \times 3,3 \times 6$ or $3 \times 9$, denoted respectively LMS-X $(3 \times 3) \sim$ LMS-X $(3 \times 9)$.

For all the tested algorithms, RS10\% varies linearly with RSU, with a unique slope, expect one. This means that the qualities of the filters are the same for all the demodulation techniques. Spectral Analysis is shifted compared to the others. This means that a local event would be rendered with a higher accuracy with this method than the others. Note that the use of a low-pass filter (LTDS-filter) translates the ultimate spatial resolutions compared to the results without this filter (LTDS). Therefore, this method has a negative influence on spatial resolution. In general, the image correlation techniques gave lower spatial resolutions than spectral techniques.

Random errors induced by all demodulation techniques are approximately $1 / 100^{\text {th }}$ pixel. Here, the implementation "LTDS-Filter" gives the best results. The correlation techniques using fewer points than the others, their resolutions are higher, in particular in the case $3 \times 3$ pixels. A final comment should be done on the comparison between LAMI and LTDS: the two implementations are based on the same windowed DFT, but the results are different: this underlines the interest on this crosscomparison work, even if the algorithms are supposed to be equivalent.

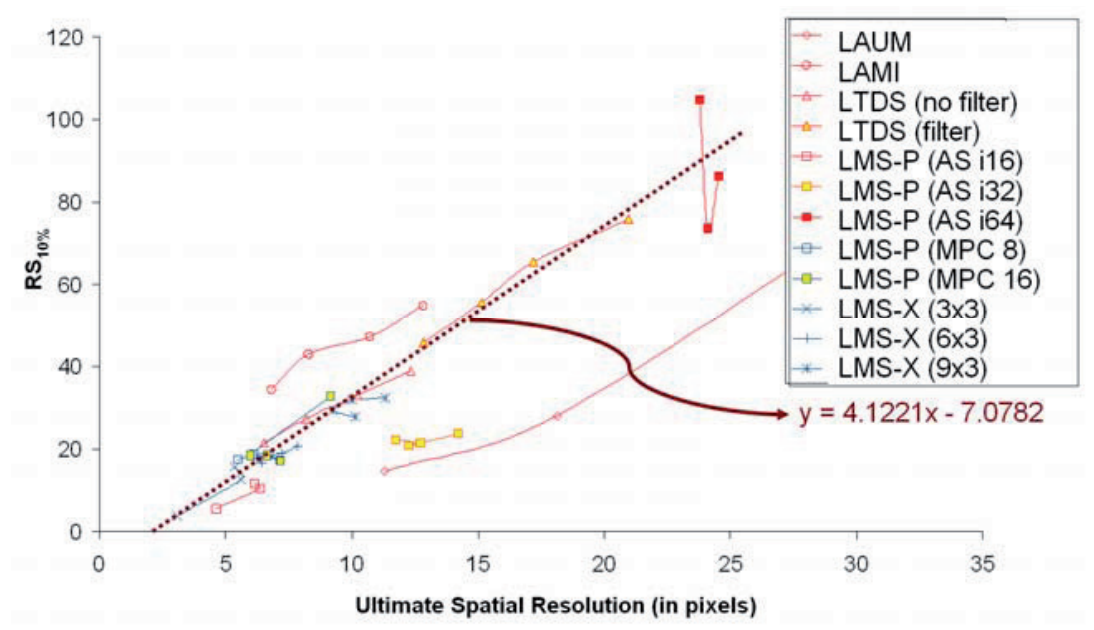

Fig. 3. $10 \%$ Spatial resolution versus ultimate spatial resolution. 


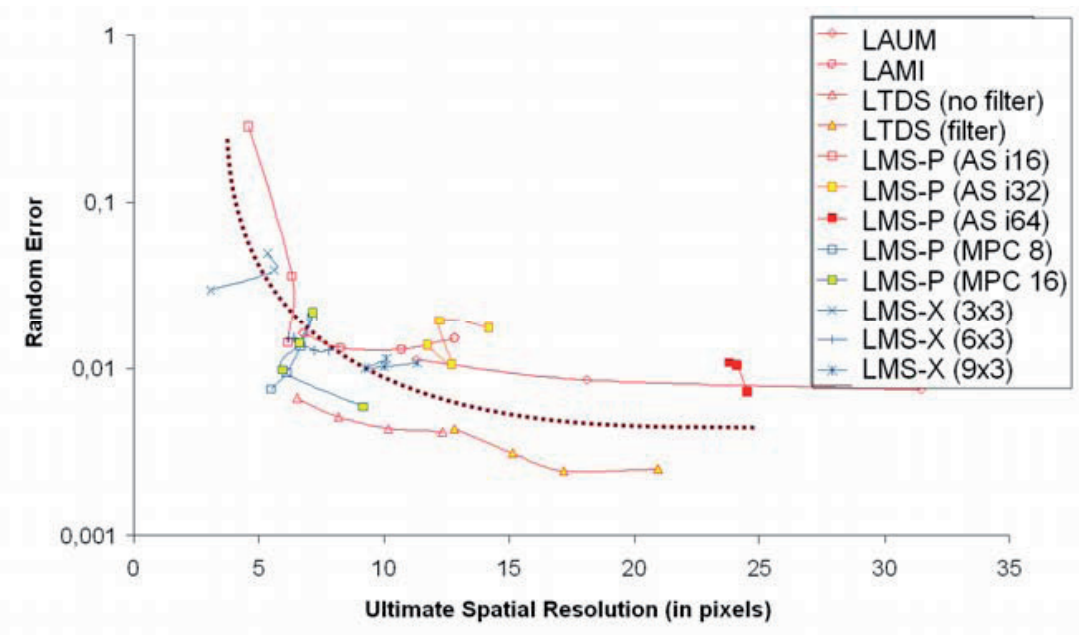

Fig. 4. Random error.

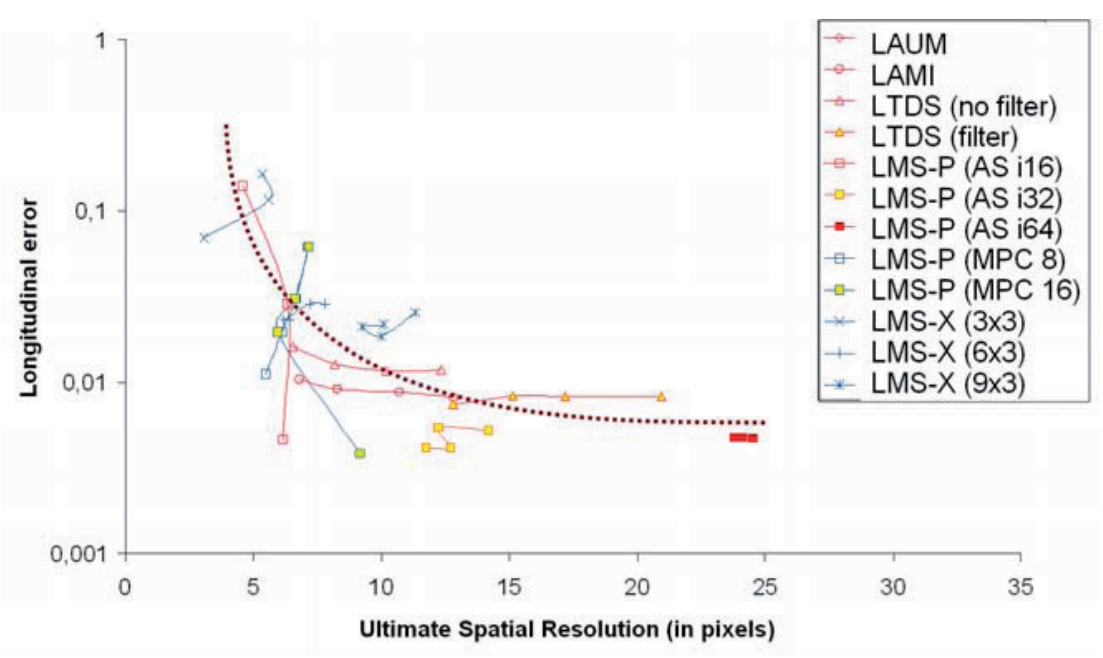

Fig. 5. Longitudinal error.

\section{Conclusion and prospects}

We proposed a new way to identify in one single step an evaluation of the main metrological quantities (bias, resolution, and spatial resolution) for an image analysis operator. Here the image analysis are fringe demodulation algorithms, based either on Fourier transform or image correlation. For this application, it has been established that the bias varies as the power of the spatial wavelength of a given event. This leads to two definitions of the spatial resolution: the first one is the detection level, defining the ultimate spatial resolution, the second one is the level corresponding to a bias lower than a threshold decided by the user (for example 10\%). It is then possible to analyse a result in function of its spatial wavelength. In future works, more representative intensity maps (additive noise, spatial variations of the contrast, different intensity encoding ...) will be used. 


\section{References}

1. M. Bornert, F. Brémand, P. Doumalin, J.-C. Dupré, M. Fazzini, M. Grédiac, F. Hild, S. Mistou, J. Molimard, J.-J. Orteu, L. Robert, Y. Surrel, P. Vacher, B. Wattrisse, Experimental Mechanics, 49, $\mathrm{N}^{\circ} 3,353,(2009)$

2. M. Takeda, H. Ina, S. Kobayashi, JOSA, 72, N¹, 156, (1982)

3. Y. Surrel, SPIE, 2342, 213, (1994)

4. P. Doumalin, M. Bornert, (P. Jacquot and J.M. Fournier Eds.), Lausanne, Springer, 67, (2000)

5. J. C. Dupré, F. Brémand, A. Lagarde, Optics and Lasers in Engineering, 18, 159, (1993)

6. D. R. Rajaona, P. Sulmont, Journal of computational Physics, 61, №1, 186, (1985)

7. E. Robin, V. Valle, Journal of Applied Optics, 43, N²2, 4355, (2004) 